\title{
NOUVELLe
}

\section{Rôle des macrophages dans le développement de l'arthrite juvénile}

\section{LACC1 fait-il le lien?}

Anne-Laure Mathieu ${ }^{1,2}$, Ommar Omarjee ${ }^{1}$, Thierry Walzer ${ }^{1,2}$, Alexandre Belot ${ }^{1,2}$

\author{
${ }^{1}$ Centre international de recherche en infectiologie, \\ U1111, CNRS UMR 5308, ENS, UCBL, 21 avenue Tony Garnier, \\ 69007 Lyon, France. \\ ${ }^{2}$ Centre de référence des rhumatismes et maladies \\ autoimmunes systémiques de l'enfant (RAISE), \\ Hôpital Femme Mère Enfant, Hospices civils de Lyon, \\ 54 boulevard Pinel, 69500, Bron, France. \\ alexandre.belot@chu-lyon.fr
}

> L'arthrite juvénile idiopathique est une maladie inflammatoire hétérogène de l'enfant caractérisée, notamment, par une atteinte articulaire. C'est le rhumatisme chronique infantile le plus fréquent, et ses causes sont encore mal connues. Sa prévalence est de 16 à 150 pour 100000 enfants en Europe et en Amérique du Nord. En France, on estime à environ 5000 le nombre d'enfants qui en sont atteints ${ }^{1}$. L'étude des rares cas familiaux de cette maladie représente une opportunité pour l'identification de nouveaux gènes impliqués, ce qui devrait permettre de progres-

1 Source : filière de santé des maladies auto-immunes et auto-inflammatoires rares: https://www.fai2r.org ser dans la physiopathologie et d'envisager des traitements plus ciblés.

\section{LACC1 : un gène impliqué dans} le développement de l'arthrite juvénile Nous avons étudié quatre familles dans lesquelles plusieurs enfants ont développé une arthrite juvénile précoce (avant l'âge de cinq ans) et sévère. Par une technique de séquençage de I'ADN à haut débit, nous avons pu identifier des mutations bialléliques du gène codant la protéine LACCl (laccase domain-containing 1) chez tous les enfants atteints [1]. Ces mutations conduisent toutes à une perte d'expression de la protéine. La fonction de LACCl, qui ne possède pas d'homologie de séquence peptidique avec d'autres protéines de mammifères, est encore assez mal connue. Dans des modèles murins, une perte d'expression de Laccl est associée à une augmentation de l'inflammation. La protéine est également impliquée dans le contrôle des infections bactériennes de l'intestin $[2,3]$. Des travaux de recherche ont par ailleurs révélé qu'elle jouait un rôle dans la transmission du signal en aval de récepteurs impliqués dans l'immunité innée [4].

Nous avons montré que LACCl est exprimée exclusivement dans les macrophages différenciés à partir de monocytes sanguins [1]. Cependant, nous n'avons pas pu mettre en évidence un rôle de la protéine 


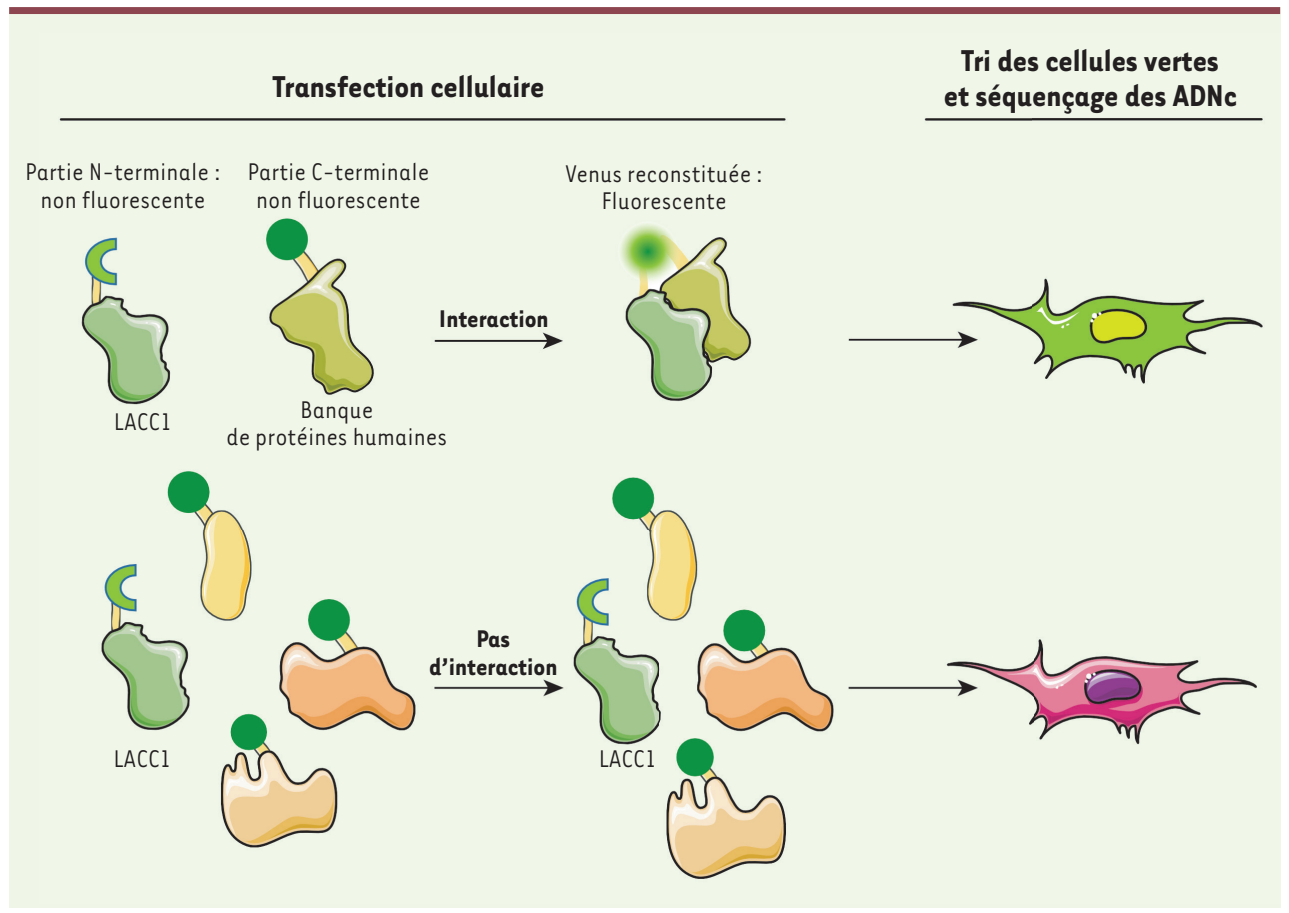

Figure 1. Principe du crible par complémentation de fluorescence. Les cellules sont co-transfectées avec un ADNc codant LACCl et une banque d'ADNc humains, chacune de ces deux constructions étant fusionnée avec l'un ou l'autre fragment de la séquence codant la protéine fluorescente Venus. Dans le cas d'une interaction protéique entre LACCl et son partenaire de liaison, les deux fragments de la protéine Venus s'assemblent, la rendant fluorescente, et les cellules fluorescentes peuvent être sélectionnées par tri cellulaire. L'ADNc codant le partenaire de liaison de LACCl est ensuite séquencé afin d'identifier la protéine qu'il code.

dans l'activation de l'une des trois voies de signalisation classiquement impliquées dans le développement des maladies inflammatoires monogéniques: la voie de l'interféron de type I (IFN-I), la voie impliquant le facteur de transcription $\mathrm{NF \kappa B}$ (nuclear factor kappa-light-chainenhancer of activated $B$ cells), et la voie de l'inflammasome [5].

\section{Identification de partenaires de LACC1 impliqués dans l'autophagie}

Nous avons donc adopté une approche non biaisée en réalisant un crible de complémentation de fluorescence afin d'identifier des partenaires de liaison de LACCl. Le principe de ce crible est de fusionner la protéine d'intérêt avec la moitié d'une protéine fluorescente (appelée Venus), et de fusionner les ADN complémentaires ( $A D N c$ ) d'une banque d'ADNc humains à une séquence codant l'autre moitié de la protéine Venus [6]. Ces deux constructions sont ensuite coexprimées dans des cellules de mammifère. Dans les cellules où LACCl interagit avec la protéine synthétisée à partir de I'ADNc qu'elles ont incorporé, la protéine Venus est reconstituée et devient fonctionnelle. Elle émet alors un signal fluo- rescent permettant de trier les cellules d'intérêt, et ainsi d'identifier les partenaires de liaison de LACCl par séquençage de I'ADNc qu'elles contiennent (Figure 1).

Cette approche nous a permis d'identifier une trentaine de partenaires de liaison potentiels de LACCl, dont RACKl (receptor of activated protein ( kinase 1 ) et AMPK (AMP-activated protein kinase), tous deux impliqués dans le contrôle de l'autophagie $[7,8]$. L'interaction de ces deux protéines avec LACCl a été confirmée par co-immunoprécipitation [1].

\section{LACC1 induit le flux autophagique dans les macrophages}

L'autophagie est un processus cellulaire de dégradation et de recyclage du contenu cytoplasmique et des membranes par les lysosomes. Elle joue un rôle essentiel dans la détoxification et le métabolisme cellulaire, et son altération peut contribuer à de nombreuses maladies [9]. Nous avons donc étudié l'autophagie dans des macrophages exprimant ou pas la protéine $\mathrm{LACCl}$, et avons observé que la formation de vésicules d'autophagie (les autophagosomes) était très diminuée en absence de $\mathrm{LACCl}$. Ce phé- notype était comparable à celui que l'on observe en absence de ATG5 (autophagyrelated 5), une protéine essentielle à la formation des autophagosomes, ce qui renforçait l'hypothèse d'un rôle de $\mathrm{LACCl}$ dans le contrôle du flux autophagique. Au contraire, une surexpression de LACCl dans des cellules $\mathrm{HeLa}^{2}$ conduit à une augmentation du nombre d'autophagosomes, donc du flux autophagique [1].

La culture des macrophages dans un milieu pauvre en nutriments induit l'autophagie, qui dépend de la phosphorylation de la protéine AMPK. Cette phosphorylation est identique en présence ou en l'absence de $\mathrm{LACCl}$, suggérant que $\mathrm{LACCl}$ intervient en aval de AMPK dans la voie de l'autophagie. Enfin, comme l'autophagie intervient également dans l'élimination des corps apoptotiques et des microorganismes pathogènes [10], nous avons mesuré la phagocytose de corps apoptotiques ou de bactéries par des macrophages exprimant ou pas $\mathrm{LACCl}$, et nous avons observé qu'elle était réduite en absence de LACCl [1].

\footnotetext{
${ }^{2}$ Ces cellules tumorales établies en une lignée utilisée par de très nombreux chercheurs proviennent d'un prélèvement tissulaire réalisé en 1951 chez une jeune femme atteinte d'un cancer métastatique du col de l'utérus.
} 


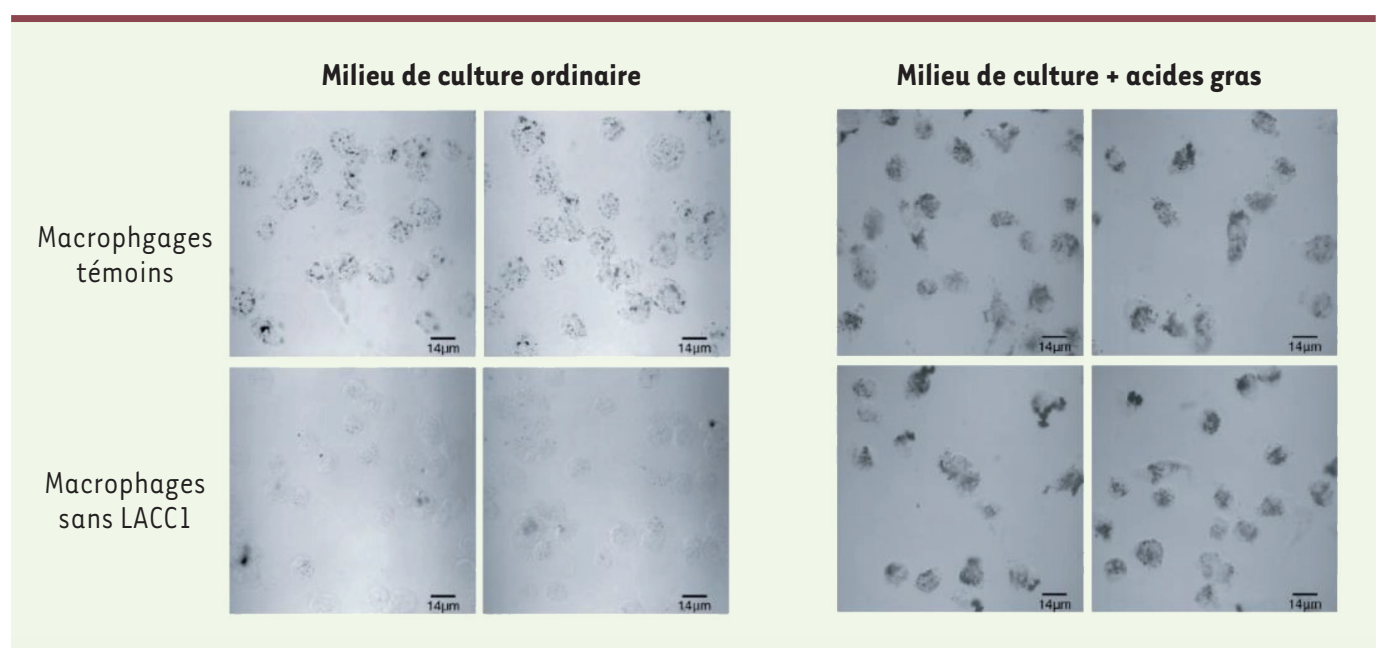

Figure 2. La protéine LACC1 favorise la production de gouttelettes lipidiques dans les macrophages. La présence de gouttelettes lipidiques a été mise en évidence à l'aide d'un colorant spécifique dans des macrophages témoins (images du haut) ou dans des macrophages dépourvus de LACCl (images du bas). Les macrophages ont été cultivés dans un milieu classique (images de gauche) ou dans un milieu supplémenté en acides gras (images de droite). En l'absence de LACCl, il n'y a pas de gouttelettes lipidiques dans le cytoplasme des macrophages, et ce défaut peut être corrigé par l'ajout exogène d'acides gras (palmitate).

\section{Rôle de LACC1 dans le métabolisme} lipidique et la respiration mitochondriale L'autophagie dépendante d'AMPK est essentielle à la production de gouttelettes lipidiques, source d'acides gras [11]. Or, une diminution de la réserve cellulaire en acides gras ainsi qu'une dérégulation du métabolisme cellulaire en absence de LACCl avaient précédemment été rapportées [12]. Nous avons donc testé l'effet de l'absence de LACCl sur la production de ces gouttelettes lipidiques, et avons observé qu'elle était très diminuée dans les macrophages qui ne synthétisaient pas LACCl (Figure 2). La production d'acétyl-CoA à partir des acides gras alimente le cycle de Krebs, donc la respiration mitochondriale, productrice d'ATP. Dans les macrophages privés de $\mathrm{LACCl}$, nous avons effectivement constaté, en mesurant leur consommation d'oxygène, une diminution de la respiration mitochondriale, qui peut être compensée par l'apport d'acides gras dans le milieu extracellulaire.

\section{Conclusion}

Nous avons mis en évidence des mutations bialléliques dans le gène codant la protéine LACCl chez plusieurs individus non apparentés atteints d'arthrite juvénile à début précoce. Cette protéine est exprimée exclusivement par les macrophages, et les mutations identifiées entraînent toutes une perte de son expression. Nous avons montré que $\mathrm{LACCl}$ est impliquée dans le contrôle de l'autophagie à travers son interaction avec des acteurs clés de ce processus. En favorisant l'autophagie dans les macrophages, LACCl pourrait permettre l'élimination de médiateurs lipidiques de l'inflammation présents dans les articulations. En effet, les gouttelettes lipidiques jouent un rôle protecteur pour la cellule en séquestrant les acides gras et en limitant l'accumulation de différents lipides cytotoxiques. Le recyclage des acides gras pourrait aussi favoriser les fonctions régulatrices des macrophages en contribuant à leur apport énergétique par la respiration mitochondriale. Le contrôle de l'autophagie étant fortement lié à la formation des lysosomes, LACCl pourrait également intervenir dans d'autres fonctions cellulaires impliquant ces organelles, notamment la présentation des antigènes aux lymphocytes. Plus généralement, un défaut de trafic membranaire en l'absence de LACCl pourrait expliquer le dysfonctionnement de l'élimination de corps apoptotiques ou de corps étrangers par phagocytose, et l'inflammation qui en résulte. $\diamond$

Macrophage involvement in juvenile arthritis onset: LACC1 bridges the gap?

\section{LIENS D'INTÉRÊT}

Les auteurs déclarent n'avoir aucun lien d'intérêt concernant les données publiées dans cet article.

\section{RÉFÉRENCES}

1. Omarjee 0 , Mathieu A-L, Quiniou G, et al. LACCI deficiency links juvenile arthritis with autophagy and metabolism in macrophages. J Exp Med $2021 ; 218$ : e20201006.

2. Kang JW, Yan J, Ranjan K, et al. Myeloid cell expression of $\mathrm{LACCl}$ is required for bacterial clearance and control of intestinal inflammation. Gastroenterology 2020 ; 159: 1051-67.

3. Skon-Hegg C, Zhang J, Wu X, et al. LACCl regulates TNF and IL-17 in mouse models of arthritis and inflammation. J Immunol 2019 ; 202 : 183-93.

4. Lahiri A, HedI M, Yan J, et al. Human LACCl increases innate receptor-induced responses and a LACCl disease-risk variant modulates these outcomes. Nat Commun $2017 ; 8$ : 15614

5. Manthiram K, Zhou Q, Aksentijevich I, et al. The monogenic autoinflammatory diseases define new pathways in human innate immunity and inflammation. Nat Immunol 2017 ; $18: 832-42$

6. Grinberg AV, Hu C-D, Kerppola TK. Visualization of Myc/Max/Mad family dimers and the competition for dimerization in living cells. Mol Cell Biol $2004 ; 24$ : 4294-308.

7. Erbil S, Oral 0 , Mitou G, et al. RACKl is an interaction partner of ATG5 and a novel regulator of autophagy.J Biol Chem 2016 ; 291 : 16753-65.

8. Zhao Y, Wang Q, Qiu G, et al. RACKl promotes autophagy by enhancing the Atgl4L-beclin 1-Vps34Vps 15 complex formation upon phosphorylation by AMPK. Cell Rep 2015 ; 13 : 1407-17.

9. Levine B, Kroemer G. Biological functions of autophagy genes: A disease perspective. Cell 2019; 176:11-42.

10. Bonilla DL, Bhattacharya A, Sha Y, et al. Autophagy regulates phagocytosis by modulating the expression of scavenger receptors. Immunity 2013 ; 39 : 537-47.

11. Nguyen TB, Louie SM, Daniele JR, et al. DGATldependent lipid droplet biogenesis protects mitochondrial function during starvation-induced autophagy. Dev Cell 2017 ; 42 : 9-21.e5.

12. Cader MZ, Boroviak K, Zhang Q, et al. Cl3orf3I (FAMIN) is a central regulator of immunometabolic function. Nat Immunol 2016 ; 17 : 1046-56. 